\title{
Asturias acogió el I curso de Derecho Animal en el Colegio de Abogados de Oviedo
}

\section{Jhésica Álvarez González}

Abogada. Miembro de la Comisión de Derecho Animal del ICAO, y de la Asociación de Letradas por el Derecho Animal y Ambiental (ALDAA)

\section{Resumen}

El Ilustre Colegio de Abogados de Oviedo, en Asturias, impartió, el pasado 14 de junio en su sede, el I Curso de Derecho Animal con seis expertos en la materia como docentes y más de un centenar de asistentes. Materias como la evolución del Derecho Animal, sus ramas penal y administrativa, las medidas cautelares a solicitar y las últimas modificaciones que afectan a la materia fueron objeto del curso, que finalizó con un éxito total.

Palabras clave: derecho animal, formación, derecho animal penal, estatuto jurídico de los animales, derecho animal administrativo sancionador, curso de derecho animal.

\section{Abstract. Asturias hosted the first Course of Animal Law at the Oviedo Bar Association}

On 14 June, at its headquarters in Asturias, the Illustrious Bar Association of Oviedo gave its first Course of Animal Law, with six experts on the subject as teachers and over a hundred assistants. Subjects such as the evolution of Animal Law, its criminal and administrative branches, requesting precautionary measures and the final modifications that affect the subject, were key to the course that ended with overall success.

Keywords: animal law, criminal animal law, course on animal law, legal status of animals, sanctioning administrative animal right 
Destacando la dedicada labor de los miembros de la Comisión de Derecho Animal y en presencia de un centenar de alumnos, Don Ignacio Cuesta Areces -Decano del Ilustre Colegio de Abogados de Oviedo-, flanqueado por Don Pedro Paulino Sánchez Sánchez Coordinador del Centro de Estudios del ICAO- y por Doña Nuria Menéndez de Llano Rodríguez -en representación de la Comisión de Derecho Animal-, dio por inaugurado el I Curso de Derecho Animal que se celebró el pasado día 14 de junio en el Ilustre Colegio de Abogados de Oviedo, Asturias.

La especialidad de la materia objeto del curso así como la absoluta novedad de su impartición en el territorio del Principado de Asturias hicieron que la recién estrenada sala de formación del Colegio de Abogados de Oviedo se llenara de asistentes expectantes, atentos y entregados. Entre la audiencia no sólo se encontraban abogados, sino que también se contó con la presencia de biólogos, veterinarios, agentes de la autoridad, miembros del SEPRONA y de distintas asociaciones protectoras de animales.

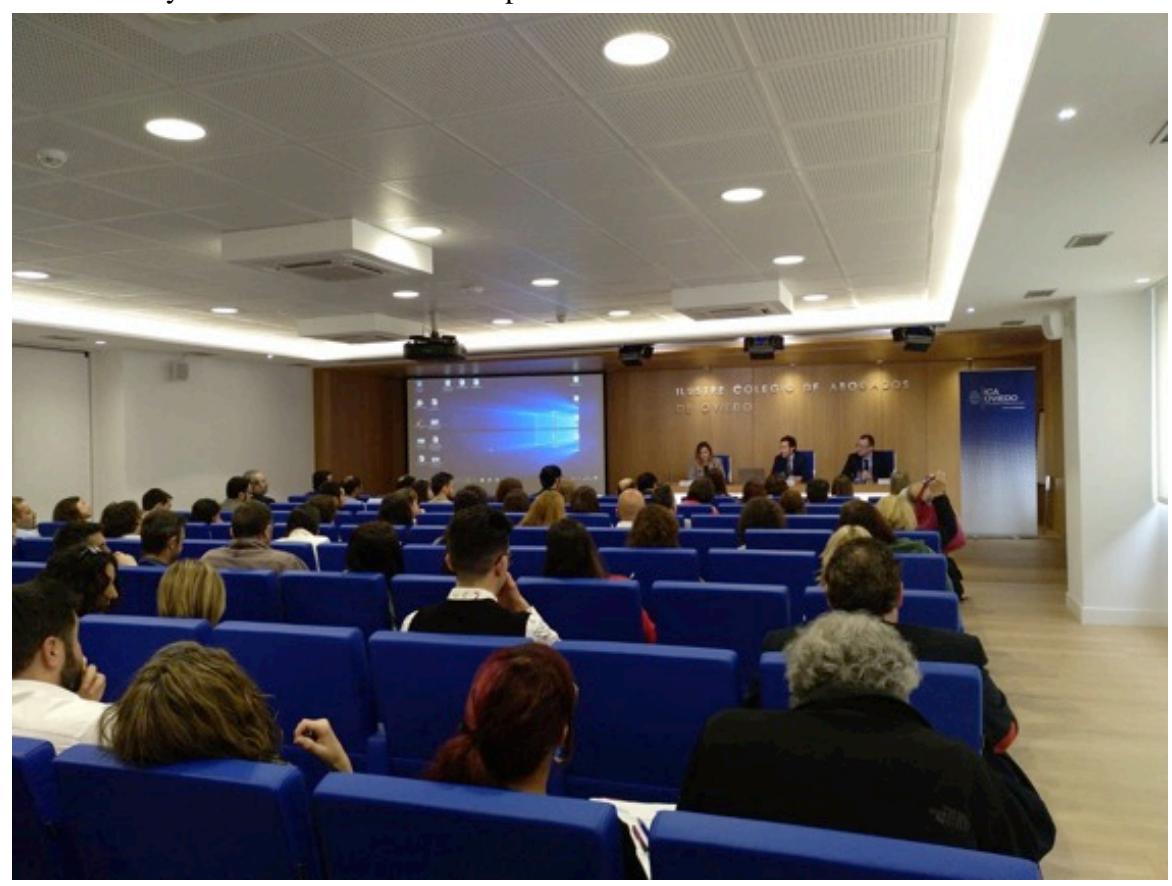

Inauguración del I Curso de Derecho Animal en el Ilustre Colegio de Abogados de Oviedo.

La Comisión de Derecho Animal del Colegio de Abogados de Oviedo se creó en el año 2017 por iniciativa de un grupo de abogados y abogadas interesados en el estudio de la materia, dispuestos a hacer cumplir sus objetivos fundacionales. Así, la citada Comisión no se plantea únicamente el estudio del Derecho Animal, sino que lucha por fomentar acuerdos institucionales en la materia, impulsa las reformas legales necesarias para la evolución del Derecho Animal en España y se compromete a difundir y formar en Derecho Animal. Dentro de este último objetivo surge la iniciativa de impartir un primer Curso de Derecho Animal dirigido tanto a colegiados como profesionales interesado en conocer más sobre las normas que regulan la situación actual de los animales. 


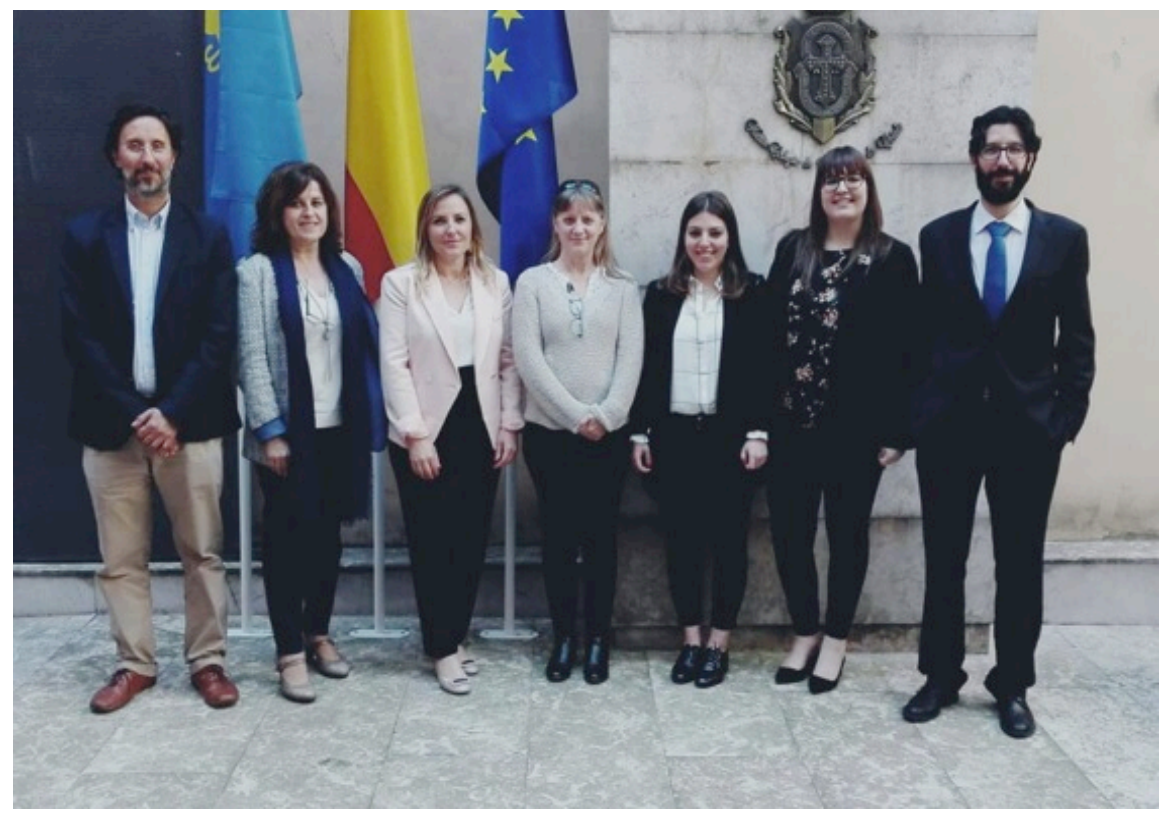

Miembros de la Comisión de Derecho Animal del ICAO tras impartir el I Curso de Derecho Animal. De izquierda a derecha: D. Benito Aláez Corral, Dña. María Jesús García Gutiérrez, Dña. Nuria Menéndez de Llano Rodríguez, Dña. Carmen Alegre Jiménez, Dña. Jhésica Álvarez González, Dña. Alicia Paredes Ramos y D. Pedro Rivas Sánchez-Arjona.

Doña Alicia Paredes Ramos ${ }^{1}$ fue la encargada de abrir el curso con su primera clase como una introducción al Derecho Animal, dedicada sentar las bases del origen del Derecho Animal, así como la evolución legislativa que da lugar al Derecho Animal vigente en España. Asimismo, se encargó de analizar los conceptos de bienestar animal y sintiencia animal como principios y cuestiones previas al estudio más pormenorizado del Derecho Animal sustantivo.

En segundo lugar, D. Benito Aláez Corral ${ }^{2}$, encargado de impartir el Derecho Animal Penal detalló la tipificación de los delitos de maltrato animal, explotación sexual animal y de abandono animal tal y como aparecen en nuestro Código Penal. También integró en su explicación lo relativo a la interposición de una denuncia penal por la comisión de los citados delitos.

Para cerrar la sesión de la mañana, Dña. Nuria Menéndez de Llano Rodríguez ${ }^{3}$ centró

\footnotetext{
${ }^{1}$ Abogada del Ilustre Colegio de Abogados de Oviedo, miembro de la Comisión de Derecho Animal del ICAO y Presidenta de la Agrupación de Letradas por el Derecho Animal y Ambiental.

${ }^{2}$ Catedrático de Derecho Constitucional de la Universidad de Oviedo, profesor e investigador universitario desde 1991, primero en la Universidad de Bochum (Alemania) hasta 1993 y desde entonces en la Universidad de Oviedo, doctor en Derecho por la Universidad de Oviedo desde 1999, ha sido premio Nacional Francisco Tomás y Valiente de Estudios Constitucionales 2003 y Magistrado suplente de la Audiencia Provincial de Asturias y del Tribunal Superior de Justicia de Asturias, también es miembro de la Comisión de Derecho Animal del ICAO, cofundador del Observatorio de Justicia y Defensa Animal y asesor de la ONG norteamericana Non Human Rights Project.

${ }^{3}$ Abogada del Ilustre Colegio de Abogados de Oviedo, doctoranda en Derecho en el Departamento de Derecho Público de la Universidad de Oviedo, colaboradora predoctoral del grupo de investigación sobre el Estatuto Jurídico de los animales de la Universidad Autónoma de Barcelona, miembro del Consejo
} 
su ponencia en poner al día a los asistentes de las principales novedades en materia de Derecho Animal que forman el nuevo marco normativo al que hemos de atenernos tras la ratificación del Convenio Europeo sobre protección de los animales de compañía. La actualización del estatuto jurídico civil de los animales (en tanto que supone la modificación del Código Civil, de la Ley Hipotecaria y de la Ley de Enjuiciamiento Civil), tras una detallada exposición del camino que supuso la consideración jurídica de que los animales no son cosas, dejó tiempo para un adelanto de las reformas en materia penal que se encuentran en tramitación en la actualidad.

Iniciando la sesión de la tarde, Dña. María Jesús García Gutiérrez ${ }^{4}$ se adentró en la materia administrativa-sancionadora haciendo un especial hincapié en la regulación de los animales considerados potencialmente peligrosos, así como la regulación nacional y autonómica del Principado de Asturias de los espectáculos con animales, desde circos hasta fiestas privadas en las que se utilizan animales como una atracción más del evento.

Continuando con el Derecho administrativo-sancionador, Dña. Jhésica Álvarez González ${ }^{5}$ expuso la segunda parte del tema, centrándose ahora en la regulación del Principado de Asturias sobre tenencia, protección y defensa de los animales, analizando la normativa vigente en la provincia así como en sus múltiples municipios. Para terminar el bloque se hizo una breve referencia a los pasos a seguir para la interposición de una denuncia por infracción de la normativa administrativa citada.

En último lugar, y cerrando así el curso, D. Pedro Rivas Sánchez-Arjona ${ }^{6}$ dedicó su exposición de forma íntegra a las medidas cautelares, tanto a las que se pueden pedir en un procedimiento penal, como en uno de carácter administrativo-sancionador. Dónde, cómo y cuándo solicitarlas fueron algunas de las cuestiones que se resolvieron en esta ponencia.

Una vez que las múltiples y, sin duda, interesantes preguntas de los asistentes quedaron resueltas por los docentes, un caluroso aplauso general dio por finalizada una jornada en la que se pueden destacar, al menos, tres aspectos fundamentales: en primer lugar, la formación en Derecho Animal no sólo es una tarea pendiente de nuestras instituciones y de un valor esencial para nuestra sociedad, sino que es una materia con una demanda cada vez más elevada, que crece con el sentir de la sociedad; en segundo lugar, precisamente este sentir y esta concienciación para con los animales, fundamenta las reformas en trámite y augura un futuro para el Derecho Animal de nuestro país en constante crecimiento y mejora, siendo cada vez más respetuoso y consciente con los animales con los que compartimos el planeta en el que vivimos; y en tercer lugar, pero no por ello menos importante, el Derecho Animal existe, y aunque su necesidad de actualización se hace evidente en algunos aspectos, nos ofrece hoy por hoy mecanismos y medidas adecuados para la lucha contra el maltrato animal y su explotación. Sólo nos resta conocerlos y ponerlos en funcionamiento de la forma más eficaz posible. Por nosotros, pero también -y sobre todo- por ellos.

Editorial de la Revista de Derecho Animal de la Universidad Autónoma de Barcelona, miembro de la Comisión de Derecho Animal del ICAO, directora del Observatorio de Justicia y Defensa Animal desde 2012 y miembro asociado del Oxford Centre for Animal Ethics.

${ }^{4}$ Abogada del ICA de Oviedo en ejercicio desde hace 26 años, miembro de la Comisión de Derecho Animal del ICAO y miembro del Patronato de la Fundación de Amigos del Perro.

${ }^{5}$ Abogada del ICA de Oviedo, doctoranda en Derecho Penal por la Universidad Complutense de Madrid, miembro de la Comisión de Derecho Animal del ICAO, y miembro de la Agrupación de Letradas por el Derecho Animal y Ambiental.

${ }^{6}$ Abogado del ICAO de Oviedo y miembro de la Comisión de Derecho Animal del ICAO. 